\title{
MISCELANEA
}

COMUNICACIÓN ORAL VII CONGRESO NACIONAL DE LA SOCIEDAD CIENTÍFICA ESPAÑOLA DE ENFERMERÍA - SCELE. Mayo de 2014. UNIVERSIDAD DE ALICANTE.

\section{CUMPLIMIENTO DE LAS RECOMENDACIONES DE APORTES DE VITAMINA D Y CALCIO EN PACIENTES CON OSTEOPOROSIS}

\section{ACHIEVEMENT OF THE RECOMMENDATIONS OF INTAKE OF VITAMIN D AND CALCIUM IN PATIENTS WITH OSTEOPOROSIS}

M Aivar Blanch, MC Mayorgas Mayorga,

JA Escamilla Fresnadillo, P Isla Pera,

J Moncho Vasallo.

CAP "Sants" Barcelona.

EUE Universidad Barcelona.

EUE Universidad Alicante.

CORREO ELECTRÓNICO: meritxellab@yahoo.es 


\section{PALABRAS CLAVE:}

Vitamina D, calcio, osteoporosis, deficiencia, incumplimiento terapéutico, menopausia.

\section{Objetivos:}

- Conocer los aportes diarios de vitamina D y calcio (suplementos farmacológicos y dieta) en mujeres mayores de 65 años.

- Conocer si existe relación entre el consumo diario de vitamina D y las variables clínicas y sociodemográficas.

\section{Métodos:}

Diseño: Estudio observacional descriptivo y transversal.

Ámbito: Centro de atención primaria urbana, CAP Sants que consta de 18 Unidades básicas asistenciales de medicina de familia.

Población: Mujeres $\geq 65$ años con el diagnóstico de osteoporosis.

Muestra: Muestra aleatoria de 385 mujeres mayores de 65 años que acuden al servicio con diagnóstico de osteoporosis (código M81). Este tamaño muestral inicial calculado para un nivel de confianza del $95 \%$ y una precisión de $+-5 \%$ podrá ser modificado en cuanto se disponga de una primera estimación de la proporción poblacional de mujeres que cumplen las recomendaciones y de la tasa de no respuesta.

Criterios de exclusión: Mujeres que tengan el diagnóstico de demencia tipo alzheimer, demencia no especificada o demencia vascular que impida realizar una entrevista adecuadamente. Pacientes con algún trastorno que afecte a la paratohormona (hiper o hipoparatiroidismo) y por lo tanto que afecte al metabolismo del calcio.

Pacientes incluidos en el programa de atención domiciliaria o institucionalizados o con alto grado de dependencia (Barthel $<40$ ).

\section{Resultados Preliminares:}

En cuanto a las variables sociales, las características de una primera muestra de 80 participantes son las siguientes: La mayoría de los sujetos tienen una percepción de su clase social de media-media $(42,5 \%)$ y media-baja $(37,5 \%)$. Sus ingresos económicos oscilan entre 401-600 euros (22,5\%) y entre 601-1000 euros (37,5\%). Sus niveles de estudios se encuentran predominantemente repartidos en nivel de primaria incompleta $(26,3)$ y EGB o similar (42,5\%). El estado civil más prevalente en la muestra es el de viuda $(42,5 \%)$, vivir solos $(41,3 \%)$ o en pareja $(33,8 \%)$. La ocupación laboral más frecuente es la de operarios/obreros semiespecializados (27,5\%) o amas de casa (25\%). El grupo de edad predominante es el comprendido entre $71-80$ años $(37,5 \%)$ seguido del de mayores de 80 años $(32,5 \%)$. La fractura más frecuente en nuestra muestra ha sido la de Colles $(17,2 \%)$ seguida de las fracturas vertebrales (13,5\%). Un $40 \%$ de los sujetos contestaron negativamente a alguna pregunta del test de Morisky Green por lo que se pueden considerar incumplidores.

El $71,3 \%$ de los participantes no llegan a las recomendaciones diarias de consumo de vitamina D de $800 \mathrm{U}$. La media de unidades día de vitamina D en la dieta ha sido de 148,52, con una mediana de 128 y una desviación estándar de 82,73 . La media de unidades día de vitamina $D$ consumida por los sujetos con los fármacos es de 358,16 con una mediana de 400 y una desviación estándar de 343,6. La media de unidades de vitamina D prescrita por los facultativos en los suplementos farmacológicos es de 429,7. Para el consumo de calcio, la media diaria de miligramos consumidos en la dieta es de 1119,19 con una mediana de 1092,5 y una desviación estándar de 376,94. El consumo real diario de calcio en los suplementos farmacológicos presenta una media de 776,37 con una mediana de 500 y una desviación estándar de 873,49 . La media de miligramos diarios de calcio prescritos por los facultativos con los suplementos farmacológicos es de 941,63 con una mediana de 915 y una desviación estándar de 960,49.

No existen diferencias estadísticamente significativas entre el consumo diario de calcio, el consumo diario de vitamina $D$ y el resto de variables analizadas. Solo en el caso del 
consumo de vitamina $\mathrm{D}$ y la edad existe una relación estadísticamente significativa en el grupo de edad entre 45-60 años, en este caso la totalidad de las participantes no llegan a las recomendaciones diarias de vitamina $D(p=0,031)$.

\section{Discusión:}

El presente estudio demuestra una elevada falta de aporte (dieta y/o fármacos) de vitamina $D$ en nuestra población de estudio (71,3\%). Tanto la dieta, los suplementos farmacológicos incluso la suma de los dos no llegan a las recomendaciones internacionales de vitamina D. Este hecho puede llevarnos a un aumento claro del riesgo de caídas y fracturas osteoporóticas con el consiguiente gasto sanitario y aumento de la morbimortalidad de los pacientes. En la bibliografía revisada el déficit de vitamina $D$ se calcula mediante determinaciones analíticas, encontrándose también un alto porcentaje de déficit de vitamina $D$ en sangre. En nuestro estudio demostramos que la causa puede ser predominantemente una ingesta insuficiente de vitamina D.

Existe un infratratamiento importante con suplementos de calcio y vitamina $D$ en nuestro centro de estudio (media VitD:358 y Calcio:941), similar al encontrado en otros estudios. Ello posiblemente sea explicado por diferentes motivos. Uno de ellos podría ser la creencia errónea de que en nuestro medio los niveles de vitamina $D$ ya son óptimos en condiciones naturales con la dieta y la exposición solar. También el hecho de que estos suplementos sean en ocasiones mal tolerados por sus problemas digestivos y mal sabor haga que el incumplimiento sea alto ( $40 \%$ en nuestro estudio). 